07

\title{
Механизм упрочнения ультрамелкозернистого алюминия после отжига
}

\author{
(C) М.Ю. Гуткин ${ }^{1,2,3}$, Т.А. Латынина ${ }^{2}$, Т.С. Орлова ${ }^{2,4}$, Н.В. Скиба ${ }^{1,3}$ \\ ${ }^{1}$ Институт проблем машиноведения РАН, \\ Санкт-Петербург, Россия \\ ${ }^{2}$ Университет ИТМО, \\ Санкт-Петербург, Россия \\ ${ }^{3}$ Санкт-Петербургский политехнический университет Петра Великого, \\ Санкт-Петербург, Россия \\ ${ }^{4}$ Физико-технический институт им. А.Ф. Иоффре РАН, \\ Санкт-Петербург, Россия \\ E-mail: m.y.gutkin@gmail.com \\ Поступила в Редакцию 16 мая 2019 г. \\ В окончательной редакции 16 мая 2019 г. \\ Принята к публикации 21 мая 2019 г.
}

\begin{abstract}
Предложена теоретическая модель, описывающая механизм упрочнения ультрамелкозернистого алюминия, полученного интенсивной пластической деформацией кручением, после низкотемпературного отжига. В рамках модели упрочнение реализуется за счет последовательной трансформации зернограничной дислокационной структуры. В частности, пластическая деформация осуществляется за счет эмиссии решеточных дислокаций из тройных стыков границ зерен, содержащих скопления зернограничных дислокаций, последующего скольжения решеточных дислокаций в теле зерна и формирования стенок переползающих зернограничных дислокаций вдоль противоположных границ зерен. Рассчитаны энергетические характеристики и критические напряжения эмиссии решеточных дислокаций. Построены теоретические зависимости напряжения течения от степени пластической деформации, демонстрирующие хорошее качественное и количественное совпадение с экспериментальными данными.
\end{abstract}

Ключевые слова: ультрамелкозернистый алюминий, упрочнение отжигом, механизмы микропластичности.

DOI: 10.21883/FTT.2019.10.48257.481

\section{1. Введение}

Хорошо известно, что большинство ультрамелкозернистых (УМЗ) материалов, полученных методами интенсивной пластической деформации (ИПД), обладают значительно более высокой прочностью по сравнению со своими крупнозернистыми аналогами [1-6]. Например, предел прочности УМЗ алюминия, полученного интенсивной пластической деформацией кручением (ИПДК), может в четыре раза превышать предел прочности крупнозернистого алюминия $[5,7,8]$.

Кроме того, недавно было обнаружено, что последующая термическая обработка (кратковременный низкотемпературный отжиг) и дополнительная пластическая деформация оказывают значительное влияние на физические и механические характеристики УМЗ металлов. Например, в работах [5,7-11] наблюдалось, что низкотемпературный отжиг приводил к существенному увеличению прочности УМЗ алюминия (Al), полученного методами ИПД. Следует отметить, что повышение прочности после отжига не характерно для крупнозернистых материалов и реализуется только в УМЗ и в нанокристаллических (НК) материалах, полученных методами ИПД, в которых большинство границ зерен (ГЗ) - большеугловые [5,7-11]. Авторы [5,7-11] отмечали, что упрочнение после отжига УМЗ Al co- провождалось значительным (до трехкратного $[5,7,8]$ ) понижением в них плотности дислокаций и переходом большеугловых ГЗ в более равновесное состояние [7-9]. Более того, в работе [12] методом дифференциальной сканирующей калориметрии было показано, что низкотемпературный отжиг Al с УМ3 структурой, полученной методом ИПДК, приводит к понижению средней удельной энергии границ зерен, то есть релаксация ГЗ при низкотемпературном отжиге была продемонстрирована экспериментально.

Согласно многочисленным экспериментальным данным $[5,7,8,10,11,13-18]$, компьютерному моделированию [19-21] и теоретическим исследованиям [22-29], ГЗ в УМЗ и НК материалах играют ключевую роль при реализации механизмов пластической деформации. Известно, в частности, что ГЗ и их тройные стыки могут служить источниками решеточных и частичных дислокаций [30,31]. Например, в экспериментальных работах [13-16] наблюдалось испускание полных и частичных дислокаций из границ зерен и их тройных стыков в УМЗ и НК алюминии и меди. Компьютерное моделирование [21] наглядно показывает, что пластическая деформация в наноструктурных материалах может происходить за счет эмиссии дислокаций из ГЗ, их скольжения в теле зерна и захвата противоположными ГЗ. В теоретических моделях [22-29] описаны меха- 
низмы эмиссии полных и частичных дислокаций из ГЗ и их тройных стыков в результате трансформации зернограничной структуры в УМЗ и НК материалах.

Отметим, что ключевая роль ГЗ в физических механизмах, определяющих прочность и пластичность УМЗ и НК материалов, отражена не только в текущей научной периодике, но и в научных монографиях, и в образовательной литературе (см., например, работы последнего десятилетия [32-36]).

Все сказанное указывает на определяющее влияние зернограничной структуры в реализации механизмов пластической деформации, ответственных за уникальные физические и механические характеристики УМЗ материалов, которые были получены методами ИПД и подвергнуты последующему отжигу. Однако сами механизмы пластической деформации, вызывающие упрочнение в результате отжига в УМЗ материалах, полученных методами ИПД, изучены пока очень слабо. На сегодня имеется только краткое описание модели упрочнения после отжига в УМЗ Al [29]. В рамках этой модели механизм пластической деформации УМЗ Al после отжига заключается в последовательной эмиссии решеточных дислокаций (РД) из тройных стыков ГЗ, их скольжения внутри зерен и последующего захвата противоположными ГЗ, причем критическое напряжение, которое контролирует безбарьерную эмиссию РД, определяется, в первую очередь, дислокационной структурой самой ГЗ, испускающей дислокации. Отжиг УМЗ материала ведет к понижению степени неравновесности ГЗ, что выражается в уменьшении числа внесенных зернограничных дислокаций (ЗГД) и, соответственно, в рамках модели [29], в снижении концентрации сдвиговых напряжений у тройных стыков ГЗ. Последнее обстоятельство требует повышения приложенного (внешнего) напряжения для эмиссии РД из тройных стыков ГЗ во внутризеренное пространство. Этим и объясняется упрочнение УМ3 Al после кратковременного отжига $[7,8,29]$.

Основная цель настоящей работы - подробное теоретическое описание механизма пластической деформации, приводящего к повышению прочности УМЗ Al, полученного методом ИПДК, путем последующего отжига. На основе модели, предложенной в работе [29], здесь дано развернутое описание методики расчета энергетических характеристик, определяющих изменение полной энергии дефектной структуры, которое соответствует реализации механизма пластической деформации УМЗ Al после отжига. При этом проведено более детальное описание геометрии рассматриваемых дислокационных структур, и внесены соответствующие поправки в проводимые расчеты. Полученные скорректированные теоретические результаты сравниваются с экспериментальными данными, изложенными в $[7,8,29]$.

\section{2. Модель}

Рассмотрим двумерную модель УМЗ материала со средним размером зерна $d$, полученного методом ИПДК и подвергнутого затем отжигу в режиме, описанном в работах $[7,8,29]$. Пусть это зерно имеет вид правильного шестиугольника (рис. 1). В исходном состоянии имеются два скопления ЗГД одинаковой мощности и разного знака, поджатых внешним сдвиговым напряжением $\tau$ к противоположным тройным стыкам ГЗ: $A$ и $B$ (рис. $1, a)$.

В рамках модели, два скопления ЗГД моделируются двумя супердислокациями $A$ и $B$ с векторами Бюргерса $\pm \mathbf{B}( \pm B$-супердислокациями) (рис. $1, a)$. Величина векторов Бюргерса $\pm B$-супердислокаций одинакова и равна $B=n_{G B D} b_{g b}$, где $n_{G B D}$ - число ЗГД в скоплении, а $b_{g b}$ - величина вектора Бюргерса ЗГД. Предполагается, что такая дефектная структура формируется в УМЗ материале, который получен методом ИПДК и подвергнут последующему отжигу.

Далее рассмотрим процесс трансформации скоплений ЗГД в тройных стыках $A$ и $B$ (рис. 1). В суммарном поле внешнего сдвигового напряжения $\tau$ и напряжений супердислокаций $A$ и $B$ происходит испускание пары РД с векторами Бюргерса $\pm \mathbf{b}( \pm \mathbf{b}-\mathrm{PД})$ из тройных стыков ГЗ $A$ и $B$ в тело зерна. Эмиссия РД происходит в результате расщепления головных дислокаций скоплений на разностные дислокации с вектором Бюргерса $\pm \mathbf{b}_{s 1}= \pm \mathbf{b} \mp \mathbf{b}_{g b}$, которые остаются в тройных стыках ГЗ $A$ и $B$, и подвижные $\pm \mathbf{b}-$ РД, которые способны двигаться внутри зерна (рис. $1, b$ ). В результате скопления ЗГД теряют головные дислокации, и величина их вектора Бюргерса $B$ уменьшается на величину $b_{g b}$, $B-b_{g b}=B_{1}$. В рамках используемого континуального подхода [30] процесс эмиссии такой пары РД состоит в появлении двух диполей $A C$ и $B D$ полных РД с векторами Бюргерса $\pm \mathbf{b}$ (рис. $1, c)$. При этом одни РД (испущенные $b$-РД $C$ и $-b$-РД $D$ ) скользят в тело зерна, а другие РД остаются у тройных стыков ГЗ $A$ и $B$ (соответственно неподвижные $-b$-РД $A$ и $b$-РД $B$ ) (рис. $1, c)$. Угол $\alpha$ задает ориентацию плоскостей скольжения РД. Для простоты в модели рассматривается симметричный случай, поэтому $b$-РД С и $-b$-РД $D$ испускаются под одинаковыми углами $\alpha$ и проходят в теле зерна одинаковое расстояние $p_{1}$, останавливаясь у противоположных ГЗ (рис. 1, $b, c)$.

Согласно модели [29] эмиссия первой пары РД становится возможной при достижении внешним сдвиговым напряжением $\tau$ некоторой критической величины $\tau_{c 1}$. При $\tau \geq \tau_{c 1} \pm b$-РД, составляющие первую пару, испускаются тройными стыками ГЗ $A$ и $B$, доходят до противоположных ГЗ, захватываются этими ГЗ и превращаются в „новые“ ЗГД (рис. $1, b-d)$. В то же время разностные $\pm b_{s 1}$-дислокации вступают в реакцию с новыми головными дислокациями скоплений ЗГД. Как следует из схемы на рис. $1, b$, модуль вектора Бюргерса разностных $\pm b_{s 1}$-дислокаций примерно равен модулю вектора Бюргерса $\pm b$-РД, который приблизительно в три раза больше модуля вектора Бюргерса зернограничных $-b_{g b}$-дислокаций. Таким образом, разностные $\pm b_{s 1}$-дислокации вступают во взаимодействие с первыми двумя головными дислокациями ЗГД скоплений, образуя 


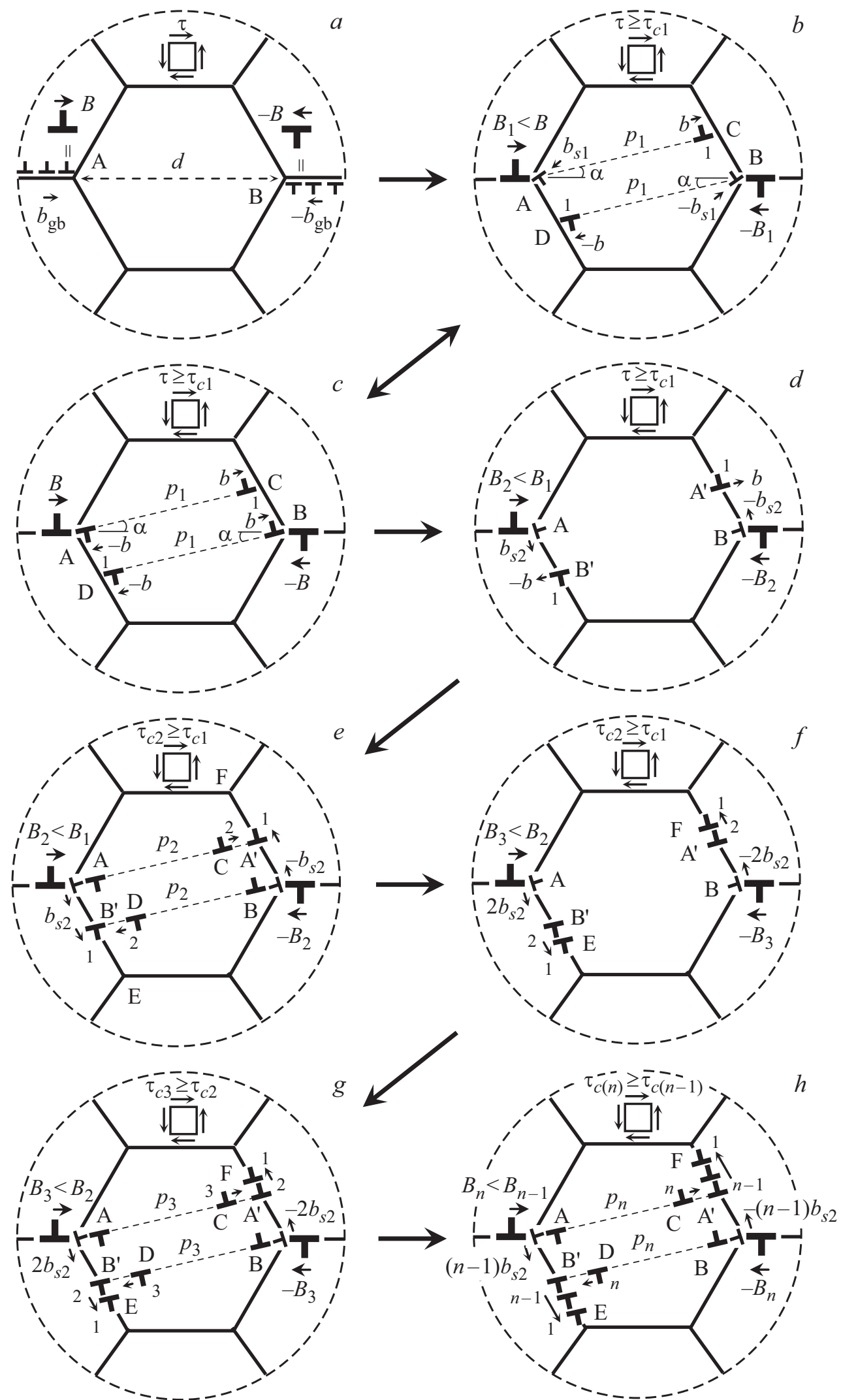

Рис. 1. Модель отдельного зерна в УМЗ материале после отжига. $a$ - исходная структура с двумя скоплениями ЗГД, которые моделируются $\pm B$-супердислокациями. $b, c-$ эмиссия первой пары РД. $d-$ захват первой пары РД границами зерен. $e-$ эмиссия второй пары РД. $f-$ переползание первой пары РД вдоль границ зерен и захват границами зерен второй пары РД. $g$ - эмиссия третьей пары РД. $h$ - эмиссия пары РД. 
новые разностные дислокации с векторами Бюргерса $\pm \mathbf{b}_{s 2}= \pm 2 \mathbf{b}_{g b} \mp \mathbf{b}_{s 1}$ (рис. $\left.1, d\right)$ и уменьшая модуль $B_{1}$ вектора Бюргерса скоплений ЗГД, $B_{1}-2 b_{g b}=B_{2}$ (рис. $1, d)$.

Далее происходит испускание второй пары $\pm b$-РД из тройных стыков ГЗ $A$ и $B$ за счет расщепления последующих головных $\pm b_{g b}$-дислокаций скоплений. Эмиссия второй пары РД также моделируется двумя диполями $\pm b$-РД: $A C$ и $B D$ (рис. $1, e)$. Эта пара РД скользит в теле зерна по тем же плоскостям скольжения, что и первая пара РД (рис. 1,e). При этом первая пара испущенных РД затрудняет эмиссию второй. Это приводит к необходимости повысить величину внешнего сдвигового напряжения $\tau$ до некоторого нового критического значения $\tau_{c 2}>\tau_{c 1}$, при котором оказывается возможным испускание второй пары $\pm b$-РД. После своего испускания эти $\pm b$-РД занимают в теле зерна некоторые положения устойчивого равновесия $p_{2}$ (рис. $1, e$ ), так как между положительными (отрицательными) РД из первой и второй пар действуют отталкивающие силы, которые не позволяют РД из второй пары пройти дальше РД из первой пары. Затем новые разностные $\pm b_{s 1}$-дислокации (которые образуются в результате дислокационных реакций, аналогичных испусканию первых РД) вступают в взаимодействие с головными $\pm b_{g b}$-дислокациями скоплений и разностными $\pm b_{s 2}$-дислокациями с образованием новых разностных дислокаций с векторами Бюргерса $\pm \mathbf{b}_{s 3}= \pm 2 \mathbf{b}_{s 2}$ (рис. $\left.1, f\right)$.

В модели предполагается, что первые РД, захваченные ГЗ $A E$ и $B F$ и превратившиеся в результате в новые ЗГД, могут перемещаться вдоль этих ГЗ путем переползания. В результате такого переползания первые $\pm b$-РД отходят от точек $A^{\prime}$ и $B^{\prime}$, в которых они были захвачены ГЗ $A E$ и $B F$, позволяя вторым $\pm b$-РД приблизиться к этим $\Gamma 3$ (рис. $1, f)$. Далее вторая пара $\pm b$-РД захватывается ГЗ $A E$ и $B F$ и трансформируется в переползающие ЗГД.

В это же время происходит испускание третьей пары $\pm b$-РД из тройных стыков ГЗ $A$ и $B$ вдоль плоскостей скольжения $A A^{\prime}$ и $B B^{\prime}$ (рис. $1, g$ ). Испускание третьих $\pm b$-РД становится возможным при достижении внешним сдвиговым напряжением критического значения $\tau_{c 3} \geq \tau_{c 2}$. Повышение $\tau$ до величины $\tau_{c 3}$ и эмиссия третьей пары РД облегчают захват ГЗ $A E$ и $B F$ второй пары РД. Третьи РД останавливаются в теле зерна в положениях своего устойчивого равновесия, пройдя расстояние $p_{3}$ (рис. $1, g$ ). При испускании последующих пар $\pm b$-РД все повторяется - предыдущие испущенные РД захватываются ГЗ $A E$ и $B F$ и превращаются в новые ЗГД, а ЗГД, образовавшиеся из ранее захваченных границами РД, продолжают переползать вдоль этих границ. При этом каждое новое испускание РД требует повышения внешнего сдвигового напряжения $\tau$ до нового критического значения $\tau_{c(n)} \geq \tau_{c(n-1)}$ (рис. $\left.1, h\right)$. В результате процессов испускания $\pm b$-РД из тройных стыков ГЗ $A$ и $B$ и последующего захвата этих РД границами $A E$ и $B F$ формируются две стенки переползающих новых ЗГД: $A^{\prime} F$ и $B^{\prime} E$ (рис. $\left.1, h\right)$.
Следует отметить, что каждое испускание РД приводит к снижению величины вектора Бюргерса $B_{n}<B_{n-1}$ скоплений ЗГД и к существенному росту критического напряжения $\tau_{c(n)}>\tau_{c(n-1)}$, которое требуется для испускания новых РД (рис. 1). Таким образом, реализация описанного механизма пластической деформации приводит к значительному упрочнению УМЗ материалов после отжига, позволяя им достигать высоких прочностных характеристик по сравнению с крупнозернистыми материалами и с УМЗ материалами, не подвергавшимися отжигу.

\section{3. Результаты}

Рассмотрим энергетические характеристики зарождения $\pm b$-РД в тройных стыках ГЗ в результате расщепления ЗГД, формирующих их исходные скопления (рис. 1). Для этого рассчитаем упругую энергию дефектной системы, находящейся под действием внешнего напряжения в различных состояниях, определяющих реализацию описанного выше механизма пластической деформации (рис. 1), с последующим сравнением полных энергий дефектной системы в этих состояниях. Трансформацию дефектной системы будем считать энергетически выгодной в случае понижения полной энергии дефектной системы при переходе из одного состояния в другое. Трансформация дефектной системы, приводящая к повышению полной энергии этой дефектной системы, является энергетически невыгодной и может быть реализована только в случае повышения уровня внешнего напряжения.

Для расчета энергетических характеристик эмиссии пар $\pm b$-РД из тройных стыков ГЗ $A$ и $B$ (рис. 2) исследуем переход дефектной системы из $(n-1)$-го состояния с полной энергией $W_{n-1}$, соответствующего образованию $(n-1)$-й пары диполей $\pm b$-РД (рис. $2, a)$, в $n$-е состояние с полной энергией $W_{n}$, соответствующее испусканию $n$-ой пары $\pm b$-РД (рис. $2, b)$. Такая трансформация дефектной системы энергетически выгодна при выполнении условия $\Delta W_{n}=W_{n}-W_{n-1}<0$.

Разность энергий $\Delta W_{n}$ определяется выражением:

$$
\Delta W_{n}=E_{\Sigma(n)}^{2 b}+E_{\Sigma(n)}^{B-2 b}+E_{(n)}^{2 b-2 b}+E_{\Sigma(n)}^{2 b-b}+E_{\Sigma(n)}^{b s-2 b}+E_{\Sigma \tau(n)},
$$

в котором используются следующие слагаемые энергии изучаемой дефектной системы, находящейся в $n$-ом состоянии: $E_{\Sigma(n)}^{2 b}-$ суммарная собственная энергия диполей $\pm b$-РД $A C$ и $B D, E_{\Sigma(n)}^{B-2 b}$ - суммарная энергия упругого взаимодействия между диполем $\pm B$-супердислокаций $A B$ и диполями $\pm b$-РД $A C$ и $B D$, $E_{(n)}^{2 b-2 b}-$ энергия упругого взаимодействия между диполями $\pm b$-РД $A C$ и $B D, E_{\Sigma(n)}^{2 b-b}-$ суммарная энергия упругого взаимодействия между диполями $\pm b$-РД $A C$ и $B D$ и стенками $A^{\prime} F$ и $B^{\prime} E$ переползающих $\pm b$-РД, $E_{\Sigma(n)}^{b s-2 b}-$ суммарная энергия упругого взаимодействия между диполями $\pm b$-РД $A C$ и $B D$ и разностными 


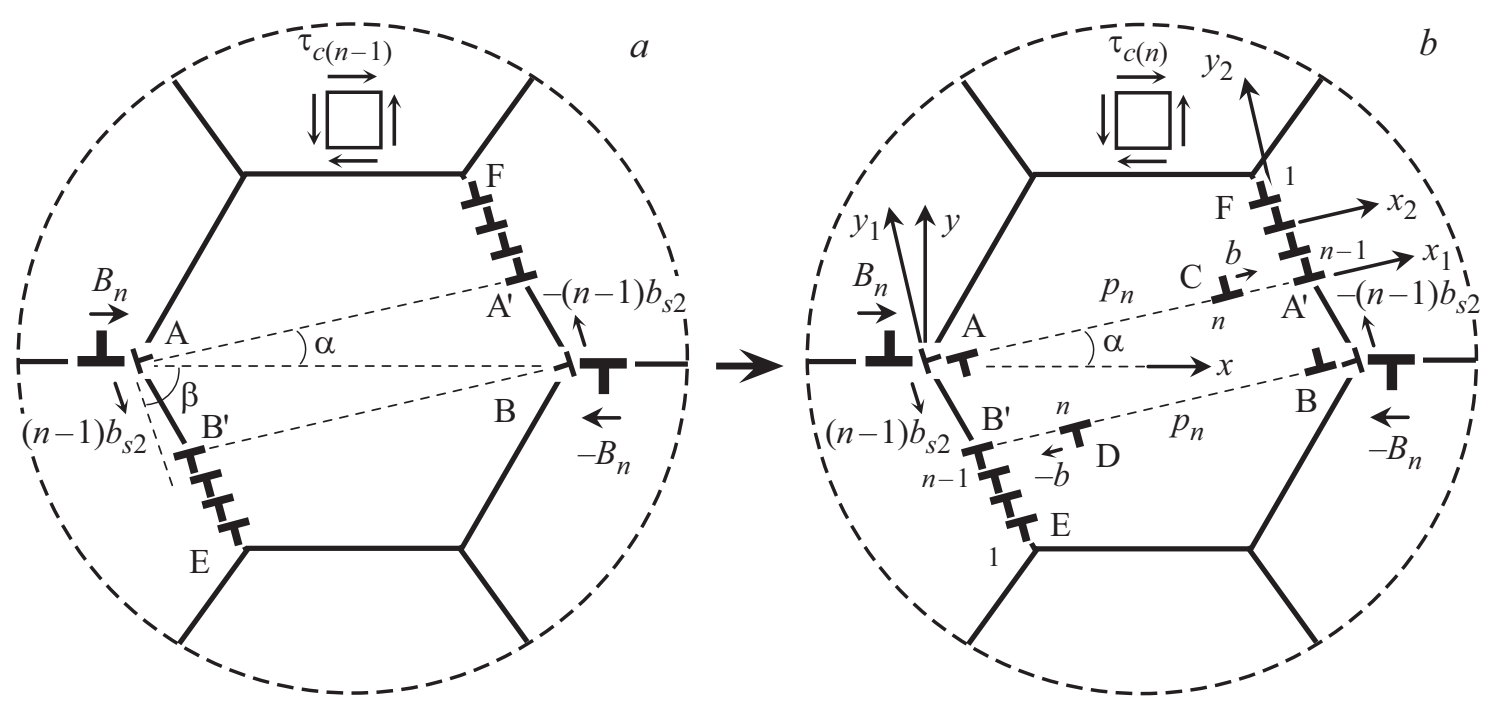

Рис. 2. Модель перехода дефектной системы из $(n-1)$-го состояния в $n$-ое состояние. $a$ - дефектная структура после образования $(n-1)$-ой пары диполей $\pm b$-РД. $b$ - дефектная структура после испускания $n$-ой пары диполей $\pm b$-РД.

$\pm(n-1) b_{s 2}$-дислокациями, $E_{\Sigma \tau(n)}-$ суммарная энергия упругого взаимодействия внешнего сдвигового напряжения $\tau$ с диполями $\pm b$-РД $A C$ и $B D$ и со стенками $A^{\prime} F$ и $B^{\prime} E$ переползающих $\pm b$-РД.

Собственные энергии $E_{(n)}^{2 b}$ диполей $\pm b$-РД $A C$ и $B D$ равны между собой, и их суммарная собственная энергия $E_{\Sigma(n)}^{2 b}$ вычисляется с помощью известного выражения [30] следующим образом:

$$
E_{\Sigma(n)}^{2 b}=2 D b^{2}\left(\ln \frac{p_{n}-r_{c}}{r_{c}}+1\right),
$$

где $D=G /[2 \pi(1-v)], G-$ модуль сдвига, $v-$ коэффициент Пуассона, $p_{n}$ - расстояние, пройденное $n$-ой решеточной $\pm b$-РД, $r_{c} \approx b-$ радиус дислокационного ядра $\pm b$-РД.

Найдем энергии $E_{\Sigma(n)}^{B-2 b}$ (в расчете на единицу длины дислокации) упругого взаимодействия между диполем $\pm B$-супердислокаций $A B$ и диполями $\pm b$-РД $A C$ и $B D$. Поскольку в модели рассматривается симметричный случай (эмиссия РД из тройных стыков $A$ и $B$ происходит симметрично, см. рис. 1$)$, энергия $E_{(n)}^{A B-A C}$ взаимодействия диполя $\pm B$-супердислокаций $A B$ с дислокационным диполем $A C$ равна энергии $E_{(n)}^{A B-B D}$ взаимодействия диполя $\pm B$-супердислокаций $A B$ с дислокационным диполем $B D$. Поэтому достаточно рассчитать только энергию $E_{(n)}^{A B-A C}$. Используя метод Муры [37], найдем ее как работу по зарождению диполя $\pm b$-РД $A C$ в поле напряжений диполя $\pm B$-супердислокаций $A B$ по следующей формуле (примеры подобных расчетов можно найти в работах $[22,24])$ :

$$
E_{(n)}^{A B-A C}=-b \int_{0}^{p_{n}} \tau_{A B}\left(x_{1}, y_{1}=0\right) d x_{1},
$$

где $\tau_{A B}\left(x_{1}, y_{1}\right)$ - сдвиговое напряжение диполя $\pm B$-супердислокаций $A B$ в плоскости $A A^{\prime}$ скольжения диполя $\pm b$-РД $A C$ (рис. $2, b)$. Компоненты тензора напряжений диполя $\pm B$-супердислокаций $A B$ в координатной системе $(x, y, z)$, в которой ось $z$ параллельна линиям дислокаций, определяются стандартными формулами [30]:

$$
\begin{aligned}
& \sigma_{x x}^{A B}=D B_{n}\left(-\frac{y\left(3 x^{2}+y^{2}\right)}{\left(x^{2}+y^{2}\right)^{2}}+\frac{y\left(3(x-d)^{2}+y^{2}\right)}{\left((x-d)^{2}+y^{2}\right)^{2}}\right) ; \\
& \sigma_{y y}^{A B}=D B_{n}\left(\frac{y\left(x^{2}-y^{2}\right)}{\left(x^{2}+y^{2}\right)^{2}}-\frac{y\left((x-d)^{2}-y^{2}\right)}{\left((x-d)^{2}+y^{2}\right)^{2}}\right) ; \\
& \sigma_{z z}^{A B}=v\left(\sigma_{x x}^{A B}+\sigma_{y y}^{A B}\right) ; \\
& \sigma_{x y}^{A B}=D B_{n}\left(\frac{x\left(x^{2}-y^{2}\right)}{\left(x^{2}+y^{2}\right)^{2}}-\frac{(x-d)\left((x-d)^{2}-y^{2}\right)}{\left((x-d)^{2}+y^{2}\right)^{2}}\right) ; \\
& \sigma_{x z}^{A B}=\sigma_{y z}^{A B}=0,
\end{aligned}
$$

где $B_{n}=\left(n_{G B D}-3 n+2\right) b_{g b}$. Согласно предложенной модели (см. раздел 2), каждое испускание РД из тройных стыков ГЗ $A$ и $B$ приводит к уменьшению модуля вектора Бюргерса $\pm B$-супердислокаций на величину $3 b_{g b}$, за исключением первого испускания, при котором величина $B$ уменьшается на $b_{g b}$. Таким образом, получаем следующий набор значений для модуля вектора Бюргерса $\pm B$-супердислокаций:

$$
\begin{aligned}
& B_{1}=B-b_{g b}=\left(n_{G B D}-1\right) b_{g b}, \\
& B_{2}=B_{1}-3 b_{g b}=\left(n_{G B D}-4\right) b_{g b}, \\
& B_{3}=B_{2}-3 b_{g b}=\left(n_{G B D}-7\right) b_{g b}, \ldots, \\
& B_{i}=\left(n_{G B D}-3 i+2\right) b_{g b},
\end{aligned}
$$


где $i=1 \ldots n$.

Компоненты поля напряжений (4) создают сдвиговое напряжение $\tau_{A B}\left(x_{1}, y_{1}\right)$ в плоскости скольжения диполя $\pm b$-РД АC вдоль оси $x_{1}$ в системе координат $\left(x_{1}, y_{1} . z_{1}\right)$, повернутой на угол $\alpha$ вокруг оси $z$ относительно системы координат $(x, y, z)$ (рис. $2, b)$ :

$$
\tau_{A B}(x, y)=\sigma_{x x}^{A B} \alpha_{1} \alpha_{2}+\sigma_{y y}^{A B} \beta_{1} \beta_{2}+\sigma_{x y}^{A B}\left(\alpha_{1} \beta_{2}+\alpha_{2} \beta_{1}\right),
$$

где $\alpha_{1}=\cos \left(x_{1}, x\right)=\cos \alpha, \beta_{1}=\cos \left(x_{1}, y\right)=\sin \alpha, \alpha_{2}=$ $=\cos \left(y_{1}, x\right)=-\sin \alpha, \beta_{2}=\cos \left(y_{1}, y\right)=\cos \alpha$.

После замены переменных $x=x_{1} \cos \alpha-y_{1} \sin \alpha$ и $y=x_{1} \sin \alpha+y_{1} \cos \alpha$ из формулы (5) получаем окончательное выражение для сдвигового напряжения $\tau_{A B}\left(x_{1}, y_{1}=0\right)$ :

$$
\begin{aligned}
& \tau_{A B}\left(x_{1}, y_{1}=0\right)=D B_{n} d \\
& \times \frac{d\left(2 x^{2}+5 d^{2}\right) \cos \alpha-x\left[2\left(x^{2}+d^{2}\right) \cos 2 \alpha+d(4 d-x \cos 3 \alpha)\right]}{2 x\left(x^{2}+d^{2}-2 d x \cos 2 \alpha\right)^{2}} .
\end{aligned}
$$

После подстановки выражения (6) в формулу (3) и интегрирования получаем

$$
\begin{aligned}
E_{(n)}^{A B-A C}= & \frac{D B_{n} b}{2}\left(\cos \alpha \ln \left[\frac{d^{2}+2 d p_{n} \cos \alpha}{p_{n}^{2}}+1\right]\right. \\
& \left.+\frac{2 d p_{n} \sin ^{2} \alpha}{d^{2}+p_{n}^{2}+2 d p_{n} \cos \alpha}\right) .
\end{aligned}
$$

Суммарная энергия $E_{\Sigma(n)}^{B-2 b}$ взаимодействия между диполем $\pm B$-супердислокаций $A B$ и диполями $\pm b$-РД $A C$ и $B D$ равна удвоенной энергии $E_{(n)}^{A B-A C}$ :

$$
E_{\Sigma(n)}^{B-2 b}=E_{(n)}^{A B-A C}+E_{(n)}^{A B-B D}=2 E_{(n)}^{A B-A C} .
$$

Аналогичным образом рассчитывается энергия $E_{(n)}^{2 b-2 b}$ упругого взаимодействия между диполями $\pm b$-РД $A C$ и $B D$, как работа по зарождению одного дислокационного диполя в поле напряжений другого. Окончательное выражение для энергии $E_{(n)}^{2 b-2 b}$ дается следующей формулой:

$$
\begin{aligned}
E_{(n)}^{2 b-2 b} & =\frac{D b^{2}}{2}\left\{\ln \frac{\left[\left(p_{n}-x_{0}\right)^{2}+y_{0}^{2}\right]\left[\left(p_{n}+x_{0}\right)^{2}+y_{0}^{2}\right]}{\left(x_{0}^{2}+y_{0}^{2}\right)^{2}}\right. \\
& \left.-\frac{4 y_{0}^{2}}{x_{0}^{2}+y_{0}^{2}}+\frac{2 y_{0}^{2}}{\left(p_{n}-x_{0}\right)^{2}+y_{0}^{2}}+\frac{2 y_{0}^{2}}{\left(p_{n}+x_{0}\right)^{2}+y_{0}^{2}}\right\},
\end{aligned}
$$

где $x_{0}=d \cos \alpha, y_{0}=d \sin \alpha$.

Далее, найдем энергию $E_{\Sigma(n)}^{2 b-b}$ упругого взаимодействия между диполями $\pm b$-РД $A C$ и $B D$, с одной стороны, и стенками переползающих $\pm b-\mathrm{PД} A^{\prime} F$ и $B^{\prime} E$, с другой стороны. Эта энергия состоит из четырех слагаемых:

$$
\begin{aligned}
E_{\Sigma(n)}^{2 b-b} & =E_{(n)}^{A B-A^{\prime} F}+E_{(n)}^{B D-B^{\prime} E}+E_{(n)}^{A C-B^{\prime} E}+E_{(n)}^{B D-A^{\prime} F} \\
& =2 E_{(n)}^{A C-A^{\prime} F}+2 E_{(n)}^{A C-B^{\prime} E},
\end{aligned}
$$

где $E_{(n)}^{A C-A^{\prime} F}$ - энергия упругого взаимодействия между диполем $\pm b$-РД $A C$ и стенкой переползающих $b$-РД $A^{\prime} F$, $E_{(n)}^{B D-B^{\prime} E}-$ энергия упругого взаимодействия между диполем $\pm b$-РД $B D$ и стенкой переползающих $-b$-РД $B^{\prime} E, E_{(n)}^{A C-B^{\prime} E}-$ энергия упругого взаимодействия между диполем $\pm b$-РД $A C$ и стенкой переползающих $-b$-РД $B^{\prime} E, E_{(n)}^{B D-A^{\prime} F}$ - энергия упругого взаимодействия между диполем $\pm b$-РД $B D$ и стенкой переползающих $b$-РД $A^{\prime} F$. В связи с симметрией дефектных структур вблизи тройных стыков ГЗ $A$ и $B$ энергии взаимодействия $E_{(n)}^{A C-A^{\prime} F}$ и $E_{(n)}^{A C-B^{\prime} E}$ соответственно равны энергиям взаимодействия $E_{(n)}^{B D-B^{\prime} E}$ и $E_{(n)}^{B D-A^{\prime} F}: E_{(n)}^{A C-A^{\prime} F}=E_{(n)}^{B D-B^{\prime} E}$ и $E_{(n)}^{A C-B^{\prime} E}=E_{(n)}^{B D-A^{\prime} F}$.

Рассмотрим энергию взаимодействия $E_{(n)}^{A C-A^{\prime} F}$. Сначала рассчитаем энергию взаимодействия диполя $\pm b$-РД $A C$ с произвольной РД из стенки переползающих $b$-РД $A^{\prime} F$. Например, энергию $E_{(n) i}^{A C-A^{\prime} F}$ взаимодействия диполя $\pm b$-РД $A C$ с $i$-ой переползающей $b$-РД, имеющей координату $\left(x_{2 i}, y_{2 i}\right)$. С помощью метода Муры [37] эта энергия рассчитывается как работа по зарождению диполя $\pm b$-РД $A C$ в поле напряжений $i$-ой переползающей $b$-РД из стенки $A^{\prime} F$ по следующей формуле:

$$
E_{(n) i}^{A C-A^{\prime} F}=-b \int_{0}^{p_{n}} \tau_{b i}^{A^{\prime} F}\left(x_{1}, y_{1}=0\right) d x_{1}
$$

где $\tau_{b i}^{A^{\prime} F}\left(x_{1}, y_{1}=0\right)$ - сдвиговое напряжение, создаваемое $i$-ой переползающей $b$-РД в плоскости $A A^{\prime}$ залегания диполя $\pm b$-РД $A C$ (рис. $2, b$ ). Компоненты тензора напряжений $i$-ой переползающей $b$-РД в координатной системе $\left(x_{2}, y_{2}, z_{2}\right)$ с осью $z_{2}$, параллельной линии РД, хорошо известны [30]:

$$
\begin{gathered}
\sigma_{x x}^{A^{\prime} F}=-D b \frac{y_{2}\left(3 x_{2}^{2}+y_{2}^{2}\right)}{\left(x_{2}^{2}+y_{2}^{2}\right)^{2}} ; \\
\sigma_{y y}^{A^{\prime} F}=D b \frac{y_{2}\left(x_{2}^{2}-y_{2}^{2}\right)}{\left(x_{2}^{2}+y_{2}^{2}\right)^{2}} ; \\
\sigma_{z z}^{A^{\prime} F}=v\left(\sigma_{x x}^{A^{\prime} F}+\sigma_{y y}^{A^{\prime} F}\right) ; \\
\sigma_{x y}^{A^{\prime} F}=D b \frac{x_{2}\left(x_{2}^{2}-y_{2}^{2}\right)}{\left(x_{2}^{2}+y_{2}^{2}\right)^{2}} \\
\sigma_{x z}^{A^{\prime} F}=\sigma_{y z}^{A^{\prime} F}=0 .
\end{gathered}
$$

Выражение для сдвигового напряжения $\tau_{b i}^{A^{\prime} F}\left(x_{1}, y_{1}=0\right) \quad$ в координатной системе $\left(x_{1}, y_{1}, z_{1}\right)$ получается подстановкой выражений (12) в формулу (5) с учетом геометрии координатных систем $\left(x_{1}, y_{1}, z_{1}\right)$ и $\left(x_{2}, y_{2}, z_{2}\right)$ и заменой переменных $x_{2}=x_{1}-x_{2 i}$ и $y_{2}=y_{1}-y_{2 i}$ (рис. $\left.2, b\right)$ в следующем виде (для $y_{1}=0$ ):

$$
\tau_{b i}^{A^{\prime} F}\left(x_{1}, y_{1}=0\right)=D b \frac{\left(x_{1}-x_{2 i}\right)\left(\left(x_{1}-x_{2 i}\right)^{2}-y_{2 i}^{2}\right)}{\left(\left(x_{1}-x_{2 i}\right)^{2}+y_{2 i}^{2}\right)^{2}} \text {. }
$$


В результате энергия взаимодействия $E_{(n) i}^{A C-A^{\prime} F}$ получается интегрированием выражения (13) с помощью формулы (11) в виде:

$$
\begin{aligned}
E_{(n) i}^{A C-A^{\prime} F}= & -D b^{2}\left(\ln \frac{\left(p_{n}-x_{2 i}\right)^{2}+y_{2 i}^{2}}{x_{2 i}^{2}+y_{2 i}^{2}}\right. \\
& \left.+\frac{2 y_{2 i}^{2} p_{n}\left(p_{n}-2 x_{2 i}\right)}{\left[\left(p_{n}-x_{2 i}\right)^{2}+y_{2 i}^{2}\right]\left(x_{2 i}^{2}+y_{2 i}^{2}\right)}\right),
\end{aligned}
$$

где $\quad x_{2 i}=p_{1}-y_{2 i} \tan \left(30^{\circ}-\alpha\right), \quad y_{2 i}=(i-1) a$, $p_{1}=d \sqrt{3} /\left[2 \cos \left(\alpha-30^{\circ}\right)\right], a-$ параметр кристаллической решетки.

Энергия взаимодействия $E_{(n)}^{A C-A^{\prime} F}$ диполя $\pm b$-РД $A C$ со всеми переползающими РД в стенке $A^{\prime} F$ получается суммированием выражения (14) по индексу $i$ от 1 до $(n-1)$ :

$$
E_{(n)}^{A C-A^{\prime} F}=\sum_{i=1}^{n-1} E_{(n) i}^{A C-A^{\prime} F} .
$$

Аналогично рассчитывается энергия взаимодействия $E_{(n)}^{A C-B^{\prime} E}$ диполя $\pm b$-РД $A C$ со стенкой $B^{\prime} E$ переползающих $-b$-РД. Окончательное выражение имеет вид:

$$
\begin{aligned}
E_{(n)}^{A C-B^{\prime} E}= & D b^{2} \sum_{i=1}^{n-1}\left(\ln \frac{\left(p_{n}-x_{3 i}\right)^{2}+y_{3 i}^{2}}{x_{3 i}^{2}+y_{3 i}^{2}}\right. \\
& \left.+\frac{2 y_{3 i}^{2} p_{n}\left(p_{n}-2 x_{3 i}\right)}{\left[\left(p_{n}-x_{3 i}\right)^{2}+y_{3 i}^{2}\right]\left(x_{3 i}^{2}+y_{3 i}^{2}\right)}\right),
\end{aligned}
$$

где $x_{3 i}=y_{3 i} \tau\left(30^{\circ}-\alpha\right), y_{3 i}=d \sin \alpha+(i-1) a$.

Подставляя теперь (14)-(16) в формулу (10), получаем выражение для суммарной энергии упругого взаимодействия $E_{\Sigma(n)}^{2 b-b}$ диполей $\pm b$-РД $A C$ и $B D$ со стенками переползающих $\pm b$-РД $A^{\prime} F$ и $B^{\prime} E$.

По аналогичной схеме выполняется расчет суммарной энергии $E_{\Sigma(n)}^{b s-2 b}$ упругого взаимодействия диполей $\pm b$-РД $A C$ и $B D$ с разностными $\pm(n-1) b_{s 2}$-дислокациями. Опуская промежуточные результаты, выпишем окончательную формулу:

$$
\begin{aligned}
& E_{\Sigma(n)}^{b s-2 b}=D b b_{s n}\{\cos (\alpha+\beta) \\
& \times\left(\ln \frac{x_{0}^{\prime \prime 2}+y_{0}^{\prime \prime 2}+p_{n}^{2}-2 p_{n} x_{0}^{\prime \prime} \cos (\alpha+\beta)-2 p_{n} y_{0}^{\prime \prime} \sin (\alpha+\beta)}{x_{0}^{\prime \prime 2}+y_{0}^{\prime \prime 2}}\right. \\
& \left.-\ln \frac{p_{n}^{2}}{b^{2}}\right)+\frac{2 y_{0}^{\prime \prime}\left[x_{0}^{\prime \prime} \sin (\alpha+\beta)-y_{0}^{\prime \prime} \cos (\alpha+\beta)\right]}{x_{0}^{\prime \prime 2}+y_{0}^{\prime \prime 2}} \\
& \left.-\frac{2\left[y_{0}^{\prime \prime}-p_{n} \sin (\alpha+\beta)\right]\left[x_{0}^{\prime \prime} \sin (\alpha+\beta)-y_{0}^{\prime \prime} \cos (\alpha+\beta)\right]}{x_{0}^{\prime \prime 2}+y_{0}^{\prime \prime 2}+p_{n}^{2}-2 p_{n} x_{0}^{\prime \prime} \cos (\alpha+\beta)-2 p_{n} y_{0}^{\prime \prime} \sin (\alpha+\beta)}\right\},
\end{aligned}
$$

где

$$
x_{0}^{\prime \prime}=d \cos \beta, \quad y_{0}^{\prime \prime}=d \sin \beta \text {; }
$$

$$
\begin{gathered}
b_{s 1}=\sqrt{b_{g b}^{2}+b^{2}-2 b_{g b} b \cos \alpha}, \\
b_{s 2}=\sqrt{4 b_{g b}^{2}+b_{s 1}^{2}-4 b_{g b} b_{s 1} \cos (\alpha+\gamma)}, \\
b_{s n}=(n-1) b_{s 2} \quad \text { при } \quad n>2, \\
\gamma=\arcsin \left(b_{g b} / b_{s 1} \sin \alpha\right), \\
\beta=\arcsin \left[b_{s 1} / b_{s 2} \sin (\alpha+\gamma)\right] .
\end{gathered}
$$

Наконец, энергия взаимодействия внешнего сдвигового напряжения $\tau$ с диполями $\pm b$-РД $A C$ и $B D$ и со стенками переползающих $\pm b$-РД $A^{\prime} F$ и $B^{\prime} E$ может быть записана следующим образом:

$$
E_{\tau(n)}=-b \tau \cos 2 \alpha\left[p_{n}+2(n-1) a\right] .
$$

Используя формулы (1)-(18), составим выражение для разности энергий $\Delta W_{n}$. Положения $p_{e q(n)}$ устойчивого равновесия испущенных $\pm b$-РД соответствуют точкам минимума на зависимостях $\Delta W_{n}\left(p_{n}\right)$ и могут быть найдены из решения уравнений $\Delta W_{n} / \partial p_{n}=0$. Выполнение условий $\Delta W_{n}\left(p_{n}=p^{\prime}\right)=0\left(\right.$ где $\left.p^{\prime}=1 \mathrm{~nm}\right),\left.\Delta W_{n}\right|_{p_{n}>p^{\prime}}<0$ и $\left.\frac{\partial \Delta W_{n}}{\partial p_{n}}\right|_{p_{n}>p^{\prime}} \leq 0$ позволяют рассчитать величину критического сдвигового напряжения $\tau_{c(n)}$, т. е. минимального сдвигового напряжения, при котором возможна эмиссия $n$-ой пары РД из тройных стыков ГЗ. Критическое напряжение $\tau_{c(n)}$ связано с напряжением течения $\sigma$ следующим образом $\sigma=2 \tau_{c(n)} / \cos 2 \alpha$.

Построим теперь зависимость напряжения течения $\sigma$ от величины сдвиговой пластической деформации $\gamma_{n}$. Пластическую деформацию $\gamma_{n}$ можно оценить с помощью следующего выражения: $\gamma_{n} \approx 2 n b / d$. Расчет зависимостей $\sigma\left(\gamma_{n}\right)$ проведем на примере УМЗ Аl, используя следующие значения параметров материала: $G=27 \mathrm{GPa}, \quad v=0.31, a=0.405 \mathrm{~nm}, \quad b=a \sqrt{2} / 2$, $b_{g b} \approx 0.1 \mathrm{~nm}$ [31]. Остальные параметры дефектной системы положим равными: $d=1000 \mathrm{~nm}$ и $\alpha=20^{\circ}$. На рис. 3 представлены результаты расчета зависимостей (сплошные кривые 1,2 и 3) для случая УМЗ алюминия при разных количествах ЗГД в скоплениях $n_{G B D}=120$ (кривая 1), 172 (2) и 250 (3). Для сравнения приведены экспериментальные кривые из работы [29] (штриховые линии) для исходного крупнозернистого технически чистого Al (Al_CG), УМ3 состояния, полученного методом ИПДК до отжига (Al_HPT) и после отжига при $150^{\circ} \mathrm{C}$ в течение $1 \mathrm{~h}\left(\mathrm{Al} \_\mathrm{HPT} \_150^{\circ} \mathrm{C}\right)$. Как видно из экспериментальных кривых Al_HPT_150 ${ }^{\circ} \mathrm{C}$, максимальное значение напряжения течения $\bar{\sigma}_{y}^{\exp } \approx 260 \mathrm{MPa}$ достигается при пластической деформации $\varepsilon^{\exp } \approx 5 \%$ (рис. 3).

На рис. 3 начальные точки теоретических зависимостей $\sigma\left(\gamma_{n}\right)$ не совпадают с началом экспериментальных кривых, так как в рамках модели теоретические зависимости $\sigma\left(\gamma_{n}\right)$ описывают напряжение течения без учета упругой составляющей. Таким образом, начальные точки теоретических кривых соответствуют напряжению и пластической деформации, при которых происходит 

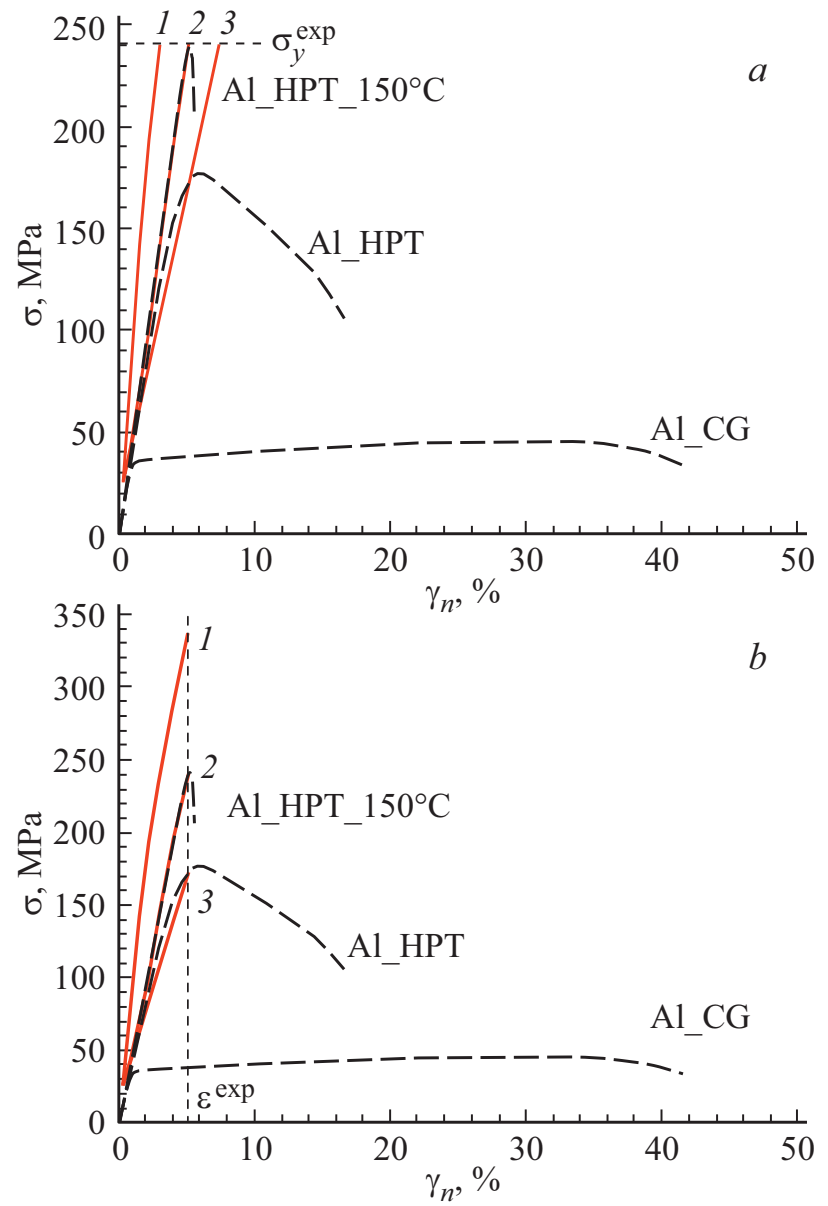

Pис. 3. Теоретические зависимости напряжения течения $\sigma$ от величины пластической деформации $\gamma_{n}$ (сплошные линии) для случая $(a) \sigma \leq \sigma_{y}^{\exp }$ и $(b) \gamma_{n} \leq \varepsilon^{\exp }$ при разном числе ЗГД в скоплениях: $n_{G B D}=120$ (кривая 1), 172 (кривая 2) и 250 (кривая 3) в сравнении с экспериментальными данными [29] (штриховые линии) для исходного крупнозернистого технически чистого Al (Al_CG), УМЗ состояния, полученного методом ИПДК до отжига (Al_HРТ) и после отжига при $150^{\circ} \mathrm{C}$ в течение $1 \mathrm{~h}$ (Al_HPT_150 $\left.{ }^{\circ} \mathrm{C}\right)$.

испускание первой пары РД. Следует также отметить, что в рамках теоретической модели отсутствуют ограничения на рост напряжения течения $\sigma$ с ростом пластической деформации $\gamma_{n}$, осуществляемой за счет испускания и скольжения РД. В связи с этим, рассмотрим два случая построения теоретических зависимостей $\sigma\left(\gamma_{n}\right)$. В первом случае ограничим рост напряжения течения $\sigma$ величиной $\sigma=\sigma_{y}^{\exp }$ (рис. $3, a$ ), при которой получим разные предельные значения пластической деформации на стадии упрочнения для разных значений $n_{G B D}$. Во втором случае наложим ограничения на рост пластической деформации $\gamma_{n}$ до величины $\gamma_{n}=\varepsilon^{\exp }$ (рис. 3,b), при которой получим разные предельные значения для напряжения течения для разных значений $n_{G B D}$.

В результате получаем, что уменьшение числа ЗГД в скоплениях приводит к снижению пластической дефор- мации $\gamma_{n}$ (рис. $3, a$ ) и к росту напряжения течения $\sigma$ в УМ3 Al (рис. 3,b). Другими словами, уменьшение в результате отжига числа ЗГД, составляющих исходные скопления, приводит к повышению прочности и уменьшению пластичности УМЗ Al. В то же время увеличение числа ЗГД в скоплениях приводит к существенному росту пластичности с уменьшением прочности, что соответствует случаю УМЗ Al, полученного методом ИПДК и не подвергавшегося термической обработке (отжигу) (рис. 3). Из сравнения теоретических зависимостей (сплошные кривые) с экспериментальными данными из работы [29] (штриховые кривые) видно, что предложенная в настоящей работе теоретическая модель дает результаты, которые хорошо соответствуют эксперименту для УМЗ Al, полученного методом ИПДК и подвергнутого последующему кратковременному низкотемпературному отжигу [29] (кривая 2 на рис. 3), а также для УМЗ Al, полученного методом ИПДК без последующего отжига (кривая 3 на рис. 3, $b$ ). Следует отметить, что предложенная теоретическая модель, объясняющая упрочнение УМЗ Al уменьшением количества дислокаций в зернограничных дислокационных скоплениях в результате релаксации зернограничной структуры при отжиге, хорошо согласуется с результатами работ [38,39]. В работе [38] методами молекулярной динамики продемонстрировано уменьшение пластичности и увеличение прочности нанокристаллического $\mathrm{Ni}$ в результате релаксации ГЗ и тройных стыков при кратковременном отжиге в более равновесное состояние. Авторы [39] экспериментально показали, что микротвердость нанокристаллического $\mathrm{Ni}$ значительно увеличивается в результате кратковременного низкотемпературного отжига, и что такое увеличение обусловлено релаксацией ГЗ.

\section{4. Заключение}

Разработана теоретическая модель, описывающая механизм упрочнения в УМ3 Al после кратковременного низкотемпературного отжига. В рамках этой модели пластическая деформация осуществляется за счет последовательной трансформации структуры ЗГД дислокаций, в результате которой происходит эмиссия РД из тройных стыков ГЗ, приводящая к формированию стенок переползающих ЗГД на противоположных ГЗ. Проведен расчет энергетических характеристик и критических напряжений эмиссии РД для УМЗ алюминия после отжига. Построены теоретические зависимости напряжения течения от величины пластической деформации, которые показали хорошее качественное и количественное совпадение с экспериментальными данными [29]. Показано, что причиной упрочнения и снижения пластичности отожженного УМЗ алюминия является снижение плотности ЗГД в неравновесных ГЗ, сформировавшихся на этапе получения УМЗ структуры методом ИПДК, в процессе отжига. 


\section{Финансирование работы}

Работа выполнена при поддержке Российского фонда фундаментальных исследований (грант № 19-08-00474). Н.В. Скиба благодарен Министерству образования и науки РФ (задание 16.3483.2017/ПЧ) за поддержку исследований.

\section{Конфликт интересов}

Авторы заявляют об отсутствии конфликта интересов.

\section{Список литературы}

[1] Y. Saito, N. Tsuji, H. Utsonomis, T. Sakai. Acta Mater. 47, 579 (1999).

[2] Z. Horita, T. Fujinami, M. Nemoto, T.G. Langdon. Met. Mater. Trans. A 31, 691 (2000).

[3] R.Z. Valiev, R.K. Islamgaliev, I.V. Alexandrov. Prog. Mater. Sci. 45, 103 (2000).

[4] J.K. Kim, H.K. Kim, J.W. Park, W.J. Kim. Scr. Mater. 53, 1207 (2005).

[5] X. Huang, N. Hansen, N. Tsuji. Science 312, 249 (2006).

[6] A. Azushima, R. Kopp, A. Korhonen, D.Y. Yang, F. Micari, G.D. Lahoti, P. Groche, J. Yanagimoto, N. Tsuji, A. Rosochowski, A. Yanagida. CIRP J. Manuf. Sci. Technol. 57, 716 (2008).

[7] A.M. Mavlyutov, A.S. Bondarenko, M.Yu. Murashkin, E.V. Boltynjuk, R.Z. Valiev, T.S. Orlova. JALCOM 698, 539 (2017).

[8] А.М. Мавлютов, Т.А. Латынина, М.Ю. Мурашкин, Р.З. Валиев, Т.С. Орлова. ФТТ 59, 1949 (2017).

[9] T.S. Orlova, A.M. Mavlyutov, A.S. Bondarenko, I.A. Kasatkin, M.Yu. Murashkin, R.Z. Valiev. Philos. Mag. 96, 2429 (2016).

[10] N. Kamikawa, X. Huang, N. Tsuji, N. Hansen. Acta Mater. 57, 4198 (2009).

[11] B.N. Semenov, I.V. Smirnov, Yu.V. Sud'enkov, N.V. Tatarinova. Mater. Phys. Mech. 24, 319 (2015).

[12] T.S. Orlova, A.V. Ankudinov, A.M. Mavlyutov, N.N. Resnina. Rev. Adv. Mater. Sci. 57, 110 (2018).

[13] M. Chen, E. Ma, K.J. Hemker, H. Sheng, Y. Wang, X. Cheng. Science 300, 5623, 1275 (2003).

[14] X.Z. Liao, F. Zhou, E.J. Lavernia, S.G. Srinivasan, M.I. Baskes, D.W. He, Y.T. Zhu. Appl. Phys. Lett. 83, 4, 632 (2003).

[15] X.Z. Liao, F. Zhou, E.J. Lavernia, D.W. He, Y.T. Zhu. Appl. Phys. Lett. 83, 24, 5062 (2003).

[16] X.Z. Liao, Y.H. Zhao, S.G. Srinivasan, Y.T. Zhu, R.Z. Valiev, D.V. Gunderov. Appl. Phys. Lett. 84, 4, 592 (2004).

[17] F. Mompiou, D. Caillard, M. Legros, H. Mughrabi. Acta Mater. 60, 3402 (2012).

[18] X. Sauvage, G. Wilde, S.V. Divinski, Z. Horita, R.Z. Valiev. Mater. Sci. Eng. A 540, 1 (2012).

[19] V. Yamakov, D. Wolf, S.R. Phillpot, A.K. Mukherjee, H. Gleiter. Nature Mater. 1, 1, 45 (2002).

[20] V. Yamakov, D. Wolf, S.R. Phillpot, H. Gleiter. Acta Mater. 50, 20, 5005 (2002).

[21] H. Van Swygenhoven. Mater. Sci. Eng. A 483-484, 33 (2008).

[22] M.Yu. Gutkin, A.L. Kolesnikova, I.A. Ovid'ko, N.V. Skiba. J. Metast. Nanocryst. Mater. 12, 47 (2002).

[23] A.A. Fedorov, M.Yu. Gutkin, I.A. Ovid'ko. Acta Mater. 51, 887 (2003)
[24] M.Yu. Gutkin, I.A. Ovid'ko, N.V. Skiba. Acta Mater. 51, 4059 (2003).

[25] М.Ю. Гуткин, И.А. Овидько, Н.В. Скиба. ФТТ 46, 11, 2042 (2004).

[26] М.Ю. Гуткин, И.А. Овидько, Н.В. Скиба. ФТТ 47, 6, 1602 (2005).

[27] M.Yu. Gutkin, I.A. Ovid'ko, N.V. Skiba. J. Phys. D 38, 3921 (2005).

[28] I.A. Ovid'ko, N.V. Skiba. Scripta Mater. 67, 1, 13 (2012).

[29] T.S. Orlova, N.V. Skiba, A.M. Mavlyutov, M.Yu. Murashkin, R.Z. Valiev, M.Yu. Gutkin. Rev. Adv. Mater. Sci. 57, 224 (2018).

[30] Дж. Хирт, И. Лоте. Теория дислокаций. Атомиздат, М. (1972). $600 \mathrm{c}$.

[31] A.P. Sutton, R.W. Balluffi. Interfaces in Crystalline Materials. Clarendon Press, Oxford (1995).

[32] А.П. Жиляев, А.И. Пшеничнюк. Сверхпластичность и границы зерен в ультрамелкозернистых материалах. Физматлит, М. (2008). 320 с.

[33] Р.А. Андриевский. Основы наноструктурного материаловедения. БИНОМ, М. (2012). 252 с.

[34] К. Коч, И. Овидько, С. Сил, С. Вепрек. Конструкционные нанокристаллические материалы. Научные основы и приложения. Физматлит, М. (2012). 448 с.

[35] Э.В. Козлов, А.М. Глезер, Н.А. Конева, Н.А. Попова, И.А. Курзина. Основы пластической деформации наноструктурных материалов. Физматлит, М. (2016). 304 с.

[36] Р.3. Валиев, А.П. Жиляев, Т.Дж. Лэнгдон. Объемные наноструктурные материалы: фундаментальные основы и применения. Эко-Вектор, СПб. (2017). 479 с.

[37] T. Mura. In: Advances in Material Research / Ed. by H. Herman. Interscience, N.Y. V. 3 (1968).

[38] A. Hasnaoui, H. Van Swygenhoven, P.M. Derlet. Acta Mater. 50, 3927 (2002).

[39] J. Hu, Y.N. Shi, X. Sauvage, G. Sha, K. Lu. Science 355, 1292 (2017).

Редактор Е.Ю. Флегонтова 